\title{
Negative Propagation Effects in Two-Dimensional Silicon Photonic Crystals
}

\author{
Ping Jiang, ${ }^{1,2}$ Kang Xie, ${ }^{2}$ Huajun Yang, ${ }^{1}$ and Zhenhai $\mathrm{Wu}^{2}$ \\ ${ }^{1}$ School of Physical Electronics, University of Electronic Science and Technology of China, Chengdu 610054, China \\ ${ }^{2}$ School of Optoelectronic Information, University of Electronic Science and Technology of China, Chengdu 610054, China
}

Correspondence should be addressed to Ping Jiang, jiangp@uestc.edu.cn

Received 31 May 2011; Revised 29 August 2011; Accepted 12 September 2011

Academic Editor: Leonid Khriachtchev

Copyright ( 2012 Ping Jiang et al. This is an open access article distributed under the Creative Commons Attribution License, which permits unrestricted use, distribution, and reproduction in any medium, provided the original work is properly cited.

\begin{abstract}
We demonstrated negative refraction effects of light propagating in two-dimensional square and hexagonal-lattice silicon photonic crystals (PhCs). The plane wave expansion method was used to solve the complex eigenvalue problems, as well as to find dispersion curves and equal-frequency contour (EFC). The finite-difference time-domain (FDTD) method was used to simulate and visualize electromagnetic wave propagation and scattering in the PhCs. Theoretical analyses and numerical simulations are presented. Two different kinds of negative refractions, namely, all-angle negative refraction (AANR) without a negative index and negative refraction with effective negative index, have been verified and compared.
\end{abstract}

\section{Introduction}

At the beginning of the 21st century, a new type of artificial material that displays anomalous properties in their interactions with electromagnetic waves has attracted a significant degree of scientific interests. These media are commonly known as "negative index materials" (NIMs) (or left-handed materials, LHMs), since its permittivities and permeabilities are simultaneously negative. NIMs can be exploited for use in a wide variety of guiding and radiating structures, such as absorber, filter, coupler, antenna, superlens, and superprism. The direction of phase velocity of light wave propagating inside such a material is opposite to the direction of its energy flow. It means that the Poynting vector and wave vector are antiparallel; therefore negative refraction occurs at the interface [1]. Veselago first pointed out the negative refraction phenomena in 1968 [2]. Years later, many theoretical and experimental groups have investigated lefthanded materials [3-5].

Since $\mathrm{PhCs}$ are more feasible to fabricate in optical region than negative index materials [6], effective-negative-index $\mathrm{PhCs}$ attracted tremendous interest of electrical engineers and physicists. One of the most prominent properties of negative index $\mathrm{PhC}$ is its capability of amplification of evanescent waves. Light propagating in such $\mathrm{PhCs}$ may exhibit negative refraction near the photonic bandgaps, such as mirror-like effect, image-transfer effect, and superprisming effect [7-12]. The effective refraction index of such $\mathrm{PhC}$, which is analogous to the effective mass in solid state physics [13], is controllable by the band structure of the material. Notomi studied negative refraction in strongly modulated PhCs near the photonic bandgaps [14]. Moussa et al. investigated superlensing behavior in $2 \mathrm{D} \mathrm{PhC}$ [15]. Cubukcu et al. demonstrated experimentally single beam negative refraction and superlensing in the valence band of 2D PhCs operating in the microwave regime [16]. Berrier et al. presented an experimental verification of negative refraction for infrared wavelength in an effective-negativeindex $\mathrm{PhC}$ [17]. Zhang proposed a 2D metal-core $\mathrm{PhC}$, which can achieve effective negative indexes for both TE and TM modes [18]. Lu et al. demonstrated an imaging experiment by using a slab of an effective-negative-index $\mathrm{PhC}$ in microwave region [19].

It was demonstrated that negative refraction phenomena in PhCs are possible in regimes of negative group velocity and negative effective index above the first band near the Brillouin zone center $\Gamma[20]$. Negative refraction can also be resulted from some special EFC in the first band of PhC [21]. The frequencies in the lowest band may be more desirable in high-resolution superlensing. 


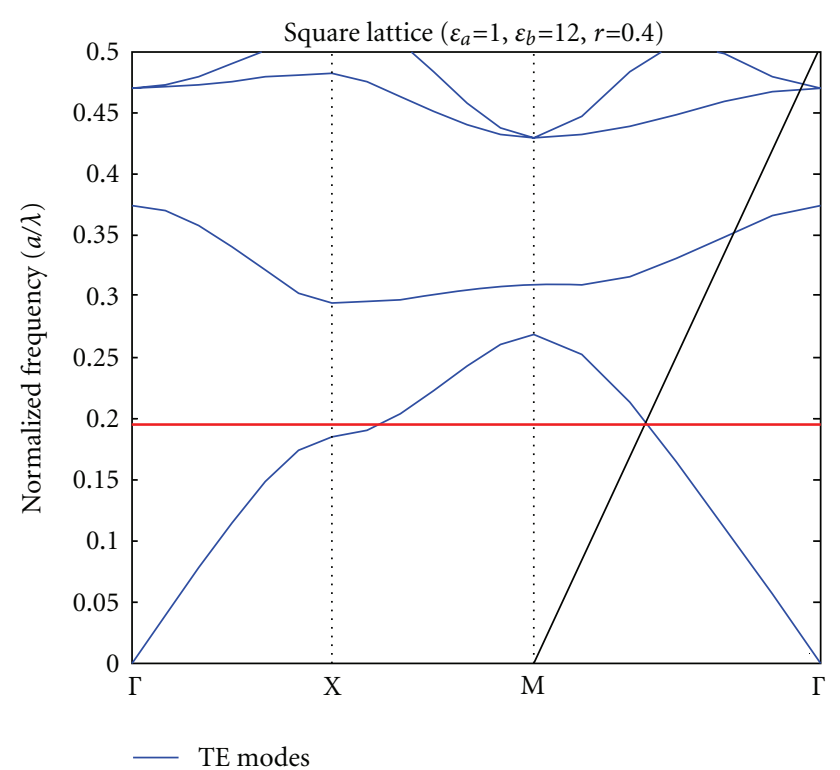

FIgUre 1: Band curves of square lattice for TE polarization. The 2D $\mathrm{PhC}$ is made of air holes of radius $r=0.4 a$ (where $a$ is the lattice constant) drilled in a silicon host substrate with $\varepsilon=12$. The light line shifted to M point is shown in black. The red line indicates the frequency range of negative refraction.

In this paper we study two different kinds of negative refraction at the first band and the second band, respectively. Firstly we study AANR without a negative index, which is resulted from a special EFC near the Brillouin zone corner $\mathrm{M}$ in the first band of a square-lattice silicon $\mathrm{PhC}$. Secondly, the negative refraction with effective negative index is investigated, which happens in regime of negative group velocity and negative effective index near the Brillouinzone center $\Gamma$ in the second band of a hexagonal-lattice silicon $\mathrm{PhC}$. The plane wave expansion method is used to solve complex eigenvalue problems. The photonic band structures and equifrequency surfaces are calculated, and two different negative refraction frequency ranges are found in two different types of PhC lattices. The finite-difference time-domain (FDTD) method is employed to simulate and visualize the subwavelength imaging effects.

\section{All-Angle Negative Refraction without a Negative Index}

In this part we consider an AANR effect that does not employ a negative effective index of refraction in the PhCs. In the first band of a square-lattice PhC, the EFC is not necessarily a circle around $\Gamma$ point, but a convexity around $\mathrm{M}$ point for certain frequency (as shown in Figure 2). So no effective refractive index can be defined in such PhCs. This kind of $\mathrm{PhCs}$ is referred to as AANR PhCs.

We begin with TE modes (in-plane electric field vector) and consider a square lattice of air holes in silicon host material with $\varepsilon=12$. The hole radius $r=0.4 a$, where $a$ is the lattice constant. Firstly, we employ the plane wave

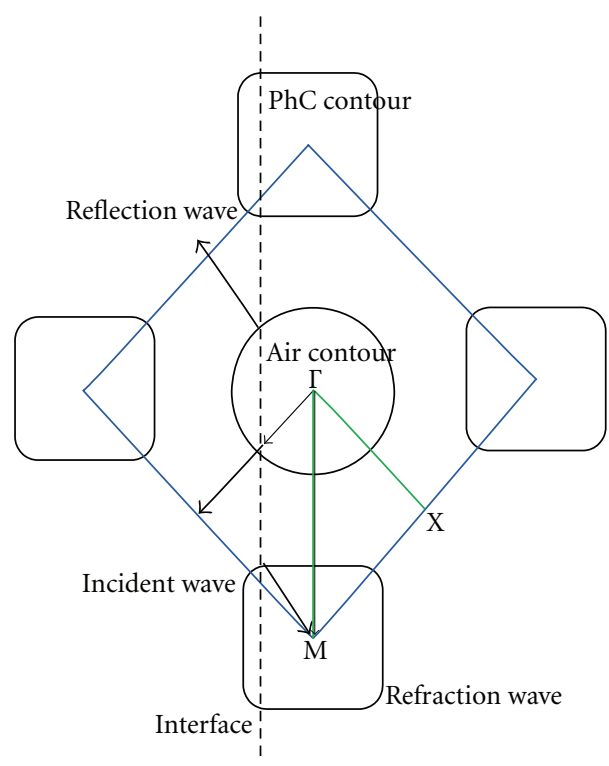

(a)

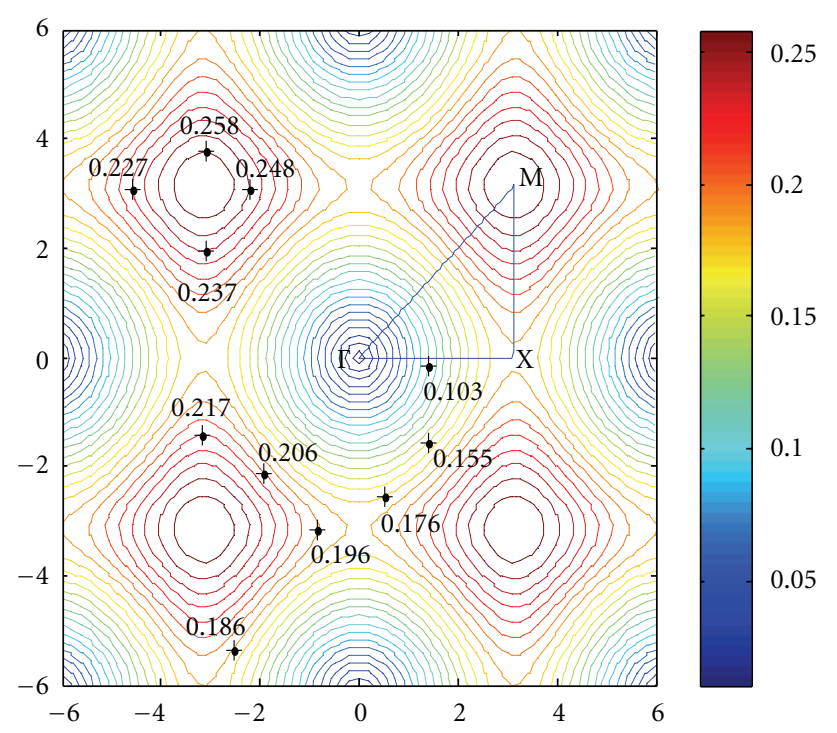

(b)

FIGURE 2: (a) Wave vector diagram of negative refraction in the $\mathrm{PhC}$. (b) EFCs of the first band of the model PhC in AANR. Value of frequency is in unit of $2 \pi c / a$.

expansion method to calculate the AANR frequency range in our model system. The band curves for TE polarization is shown in Figure 1. It is identified that the AANR region occurs in the vicinity of $\omega=0.211(2 \pi c / a)$. The upper frequency limit is determined directly by the intersection of the first band curve with the light line. The lower frequency limit is determined by the frequency at which radius of the curvature of the contours along ГМ becomes divergent (as shown in Figure 2(b)).

The contours of dispersion of the first band, drawn in the first Brillouin zone, are shown in Figure 2(a), where the thick arrows represent directions of group velocity, and thin 


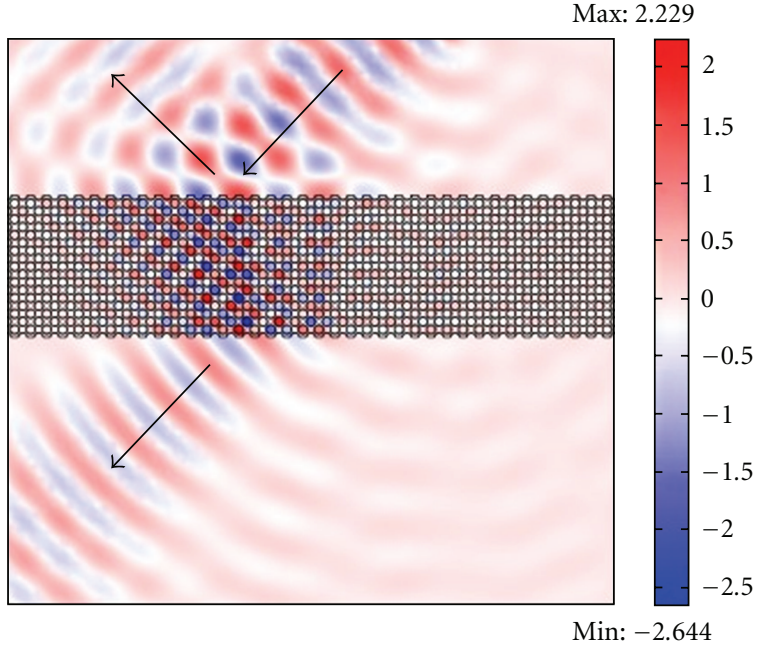

Figure 3: The negative refraction phenomena of $\mathrm{PhC}$.

arrows stand for directions of phase velocity. The EFCs of the actual $\mathrm{PhC}$ are shown in Figure 2(b). AANR occurs at frequencies that correspond to all-convex contours with a negative photonic effective mass. If the air-PhC interface normal is along the $\Gamma-\mathrm{M}$ direction, and the contour is everywhere convex, an incoming plane wave from air will couple to a single mode that propagates in this $\mathrm{PhC}$ on the negative side of the boundary normal.

Figure 3 shows the negative refraction of a light beam across a square-lattice $2 \mathrm{D} \mathrm{PhC}$ slab. The incoming wave with normalized frequency of $\omega=0.211(2 \pi c / a)$ is incident from up right side with an angle of $45^{\circ}$ with respect to the normal (as shown by the arrows). The refracted light inside the slab is on the right side of the normal. On its arrival at the lower surface of the slab, negative refraction happens again. The outgoing beam has the same propagation direction as the incoming beam but with a negative transverse shift.

AANR is essential for superlensing applications. The existence of AANR effect implies that there is essentially no physical limit on aperture of the imaging system. Such a superlens possesses several key advantages over conventional lenses. To realize AANR for superlensing, the required conditions for our model system are that the EFCs should be both convex and larger than the EFCs for air (circles with radius $\omega / \mathrm{c}$ ). Incident beams at any incident angle will then experience negative refraction when entering the $\mathrm{PhC}$. Thickness and finishing of the surfaces of the $\mathrm{PhC}$ slab should be chosen carefully to minimize reflections. Moreover, when air holes (or dielectric rods) are cut in half at the terminating surfaces, surface mode can come to exist on the slab. The transmission of evanescent waves can be amplified through the excitation of surface modes in a slab of $\mathrm{PhC}$, which plays a very important role in subwavelength imaging.

Shown in Figure 4 are the FDTD simulations of a continuous-wave point source in front of a $\mathrm{PhC}$ slab of square lattice. The radiation is TE polarized at frequency $\omega=$ $0.211(2 \pi c / a)$. The slabs in Figures 4(a)-4(c) are, respectively,

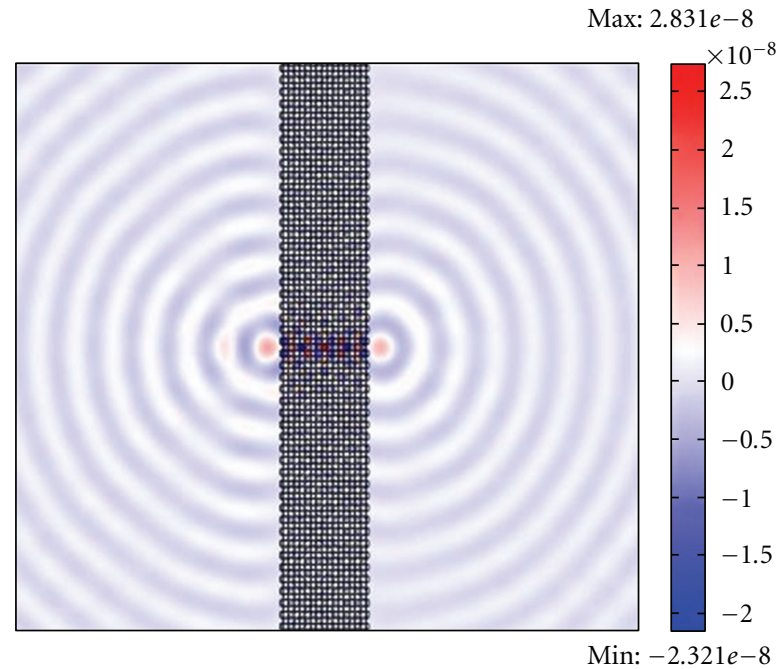

(a)

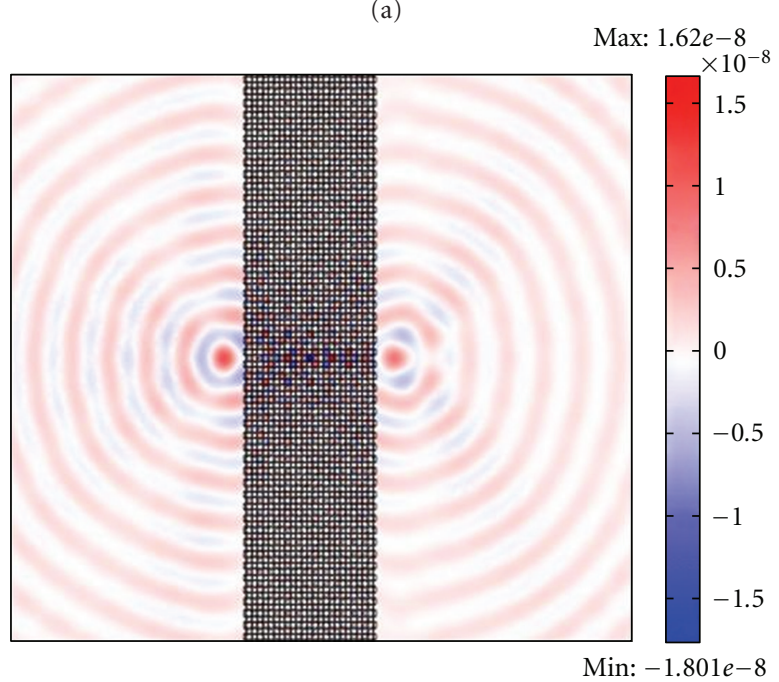

(b)

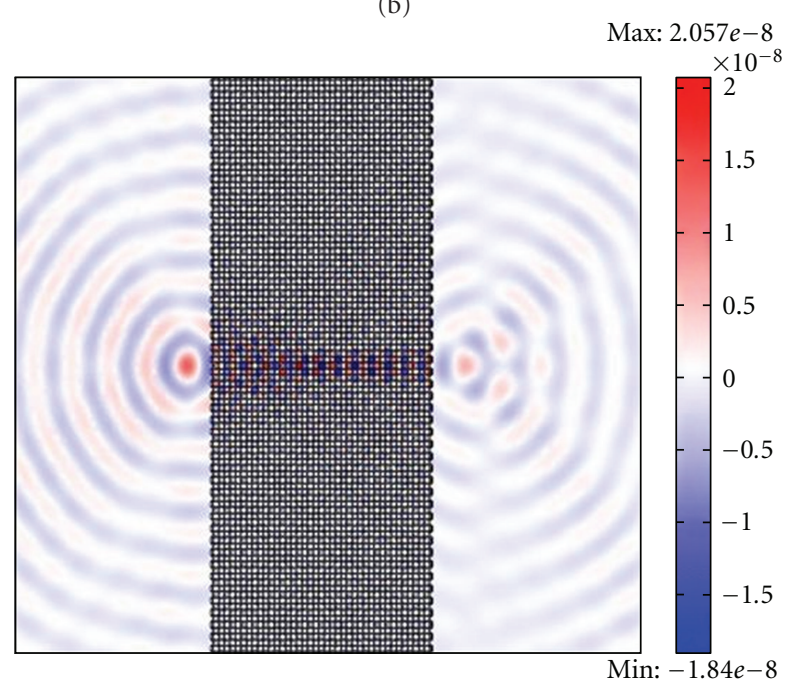

(c)

FIGURE 4: TE polarization of a point source and its image across a $\mathrm{PhC}$ slab of square lattice. The normalized frequency is $\omega=$ $0.211(2 \pi c / a)$. (a) 8-layer PhC slab, (b) 12-layer PhC slab, and (c) 20-layer PhC slab. 


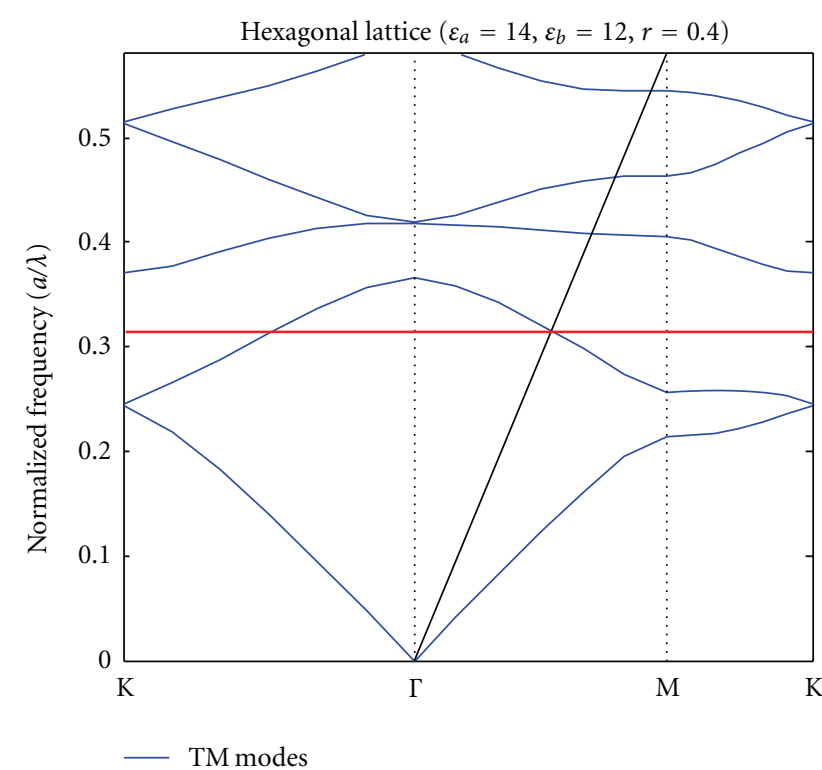

Figure 5: Band curves of 2D PhC of hexagonal lattice for TM polarization. The lattices are made of air holes of radius $r=0.4 a$ in a silicon host material with $\varepsilon=12$. The light line shifted to $\Gamma$ point is shown in black. The red line indicates the frequency range of negative refraction.

8 layers, 12 layers, and 20 layers thick. The distances of point sources to the left-hand side surfaces of the slabs are respectively, $1.5 a, 2 a$, and $3 a$. It is seen that an image of the point source is formed in each case at, respectively, a distance of $1.5 a, 2 a$, and $3 a$ away from the right-hand side surface of the superlens. Although small aberrations are visible in the field patterns, the simulations clearly demonstrate that superlensing effect occurs in these $\mathrm{PhC}$ slab systems. An infinitely long slab can focus all the Fourier components of a subject within this frequency range (including both propagation waves and evanescent waves), so that to produce a perfect image [22]. The flat lens property of AANR PhCs has many important potential applications, such as highcapacity optical storage systems [23].

\section{Negative Refraction with Effective Negative Index}

Distinguished from AANR PhCs, there is another kind of $\mathrm{PhCs}$ that can also give rise to negative refraction. It is referred to as effective-negative-index PhCs. In this part we consider the negative refraction property of $\mathrm{PhC}$ of $2 \mathrm{D}$ hexagonal lattices that are made of air holes. The background material is silicon $(\varepsilon=12)$, and the radius of the air holes is $0.4 a$, where $a$ is the lattice constant. The band curves for TM polarization (in-plane magnetic field vector) are shown in Figure 5. A negative refraction region is identified in the vicinity of $\omega=0.31(2 \pi c / a)$ where the second band curve and the light line intersect.

Shown in Figure 6 are EFCs of TM mode for the normal frequency range between $\omega=0.260(2 \pi c / a)$ and $\omega=$

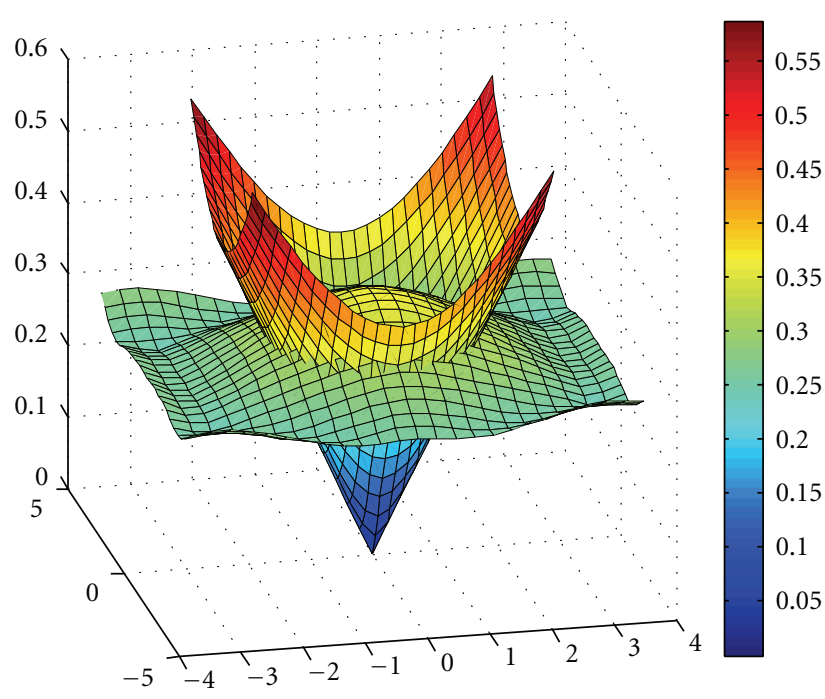

(a)

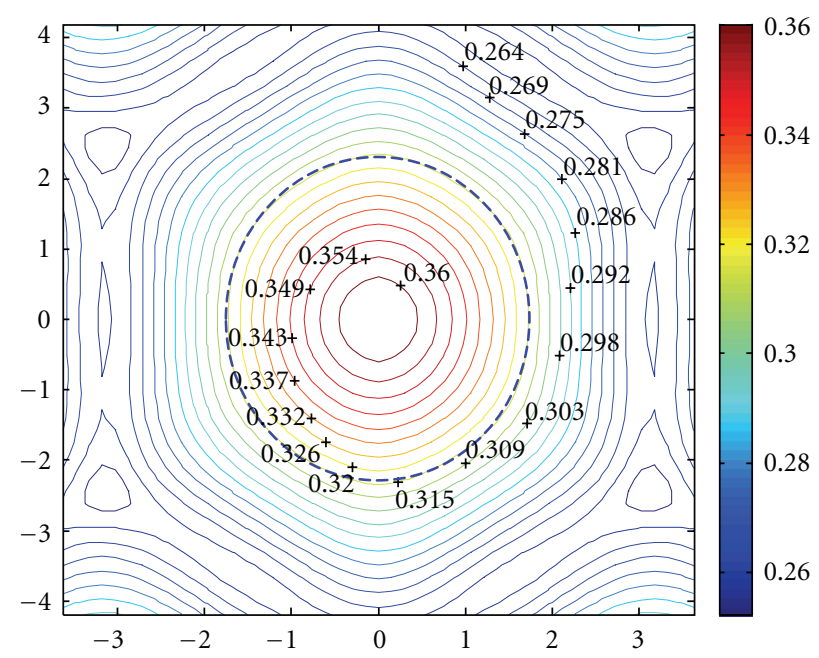

(b)

Figure 6: Band structure and EFCs of the second band of the 2D $\mathrm{PhC}$ of hexagonal lattice for TM polarization. Values of frequency are in units of $2 \pi c / a$. (a) Surfaces of the second band and light cone. (b) EFCs of the 2nd band. The blue dotted circle is the line of intersection of the second band surface and the light cone.

$0.360(2 \pi c / a)$. The shapes of EFCs for the second band are almost circular in the frequency range between $\omega=$ $0.300(2 \pi c / a)$ and $\omega=0.360(2 \pi c / a)$, where higher frequency contours concentrate near the $\Gamma$ point (the center of circles). The blue dotted circle is the line of intersection of the second band surface and the light cone. The radius of the dotted circle is equal to the radius of $\mathrm{EFC}$ of air cone at $\omega=$ $0.315(2 \pi c / a)$.

The EFC of frequency $\omega=0.315(2 \pi c / a)$ is plotted in Figure 7. Light of this frequency is incident from air $(n=1)$ into the model $\mathrm{PhC}$ that has an effective negative index $n_{\text {eff }}$ (as shown in Figure 7). In the PhC, the energy velocity vector coincides with the group velocity vector of the Bloch mode 


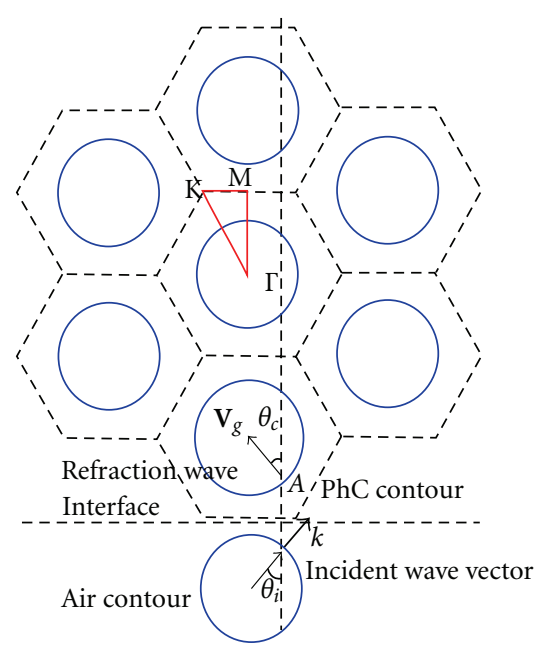

Figure 7: The EFC of frequency $\omega=0.315(2 \pi c / a)$. The air-PhC interface is normal to the $\Gamma-\mathrm{M}$ direction. $\theta_{i}$ is the incident angle of the plane wave. A Bloch wave denoted by A is excited in the PhC. $v_{g}$ is the group velocity of the Bloch wave, which has a refraction angle of $\theta_{c}$ against the normal of the interface.

$V_{g}=\nabla_{k} \omega$. A Bloch mode denoted by A is excited in the $\mathrm{PhC}$ along the direction perpendicular to the EFC and point to $\Gamma$. Given the conservation of tangential wave vector, negative refraction occurs at the interface between air and $\mathrm{PhC}$.

Using Snell's law, light propagation satisfies

$$
\frac{\sin \theta_{i}}{\sin \theta_{c}}=n_{\mathrm{eff}},
$$

where $\theta_{i}$ is the incident angle in air and $\theta_{c}$ is the refraction angle in the PhC. Because of the negative refraction, $\theta_{c}<0$ which leads to $n_{\text {eff }}<0$. It can be inferred that the absolute value of $n_{\text {eff }}$ approximates to the ratio of the radius of the $\mathrm{PhC}$ contour to the radius of the air contour. For the frequency $\omega=0.315(2 \pi c / a)$, the radius of the $\mathrm{PhC}$ contour equals the radius of the air contour (represented by the blue dotted line in Figure 6(b)), and the effective negative index is therefore $n_{\mathrm{eff}}=-1$. It can be derived that when frequency is higher than $\omega=0.315(2 \pi c / a), n_{\mathrm{eff}}>-1$. For frequency lower than $\omega=0.315(2 \pi c / a), n_{\text {eff }}<-1$. The effective negative index $n_{\text {eff }}$ of the $\mathrm{PhC}$ varies with the working frequency.

The homogeneity of refractive index of a $\mathrm{PhC}$ with effective negative index can be judged by its property of imaging as a flat lens. An isotropic homogeneous medium with refractive index of $n=-1$ creates an image of a point source on the other side of the slab at a distance which depends on the distance of the source to the slab. The relation between source distance and image distance is illustrated directly from the simple ray diagram in Figure 8. There is no reflection at the interface between a negative index material of $n=-1$ and air $(n=1)$. The coupling coefficient for any incident angle is always $100 \%$. On the other hand, for a PhC with effective negative index of $n_{\mathrm{eff}}=-1$, the situation is slightly different. The FDTD simulations of

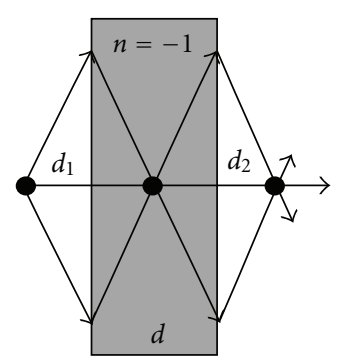

(a)

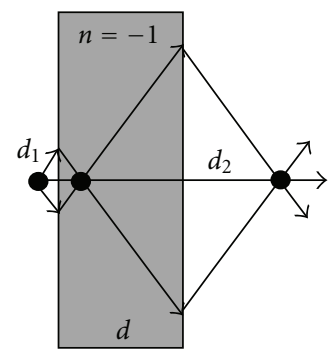

(c)

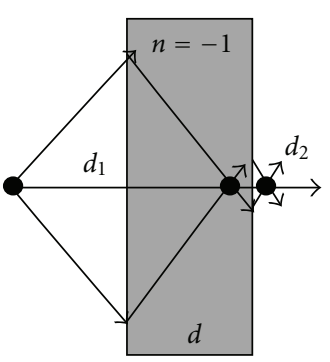

(b)

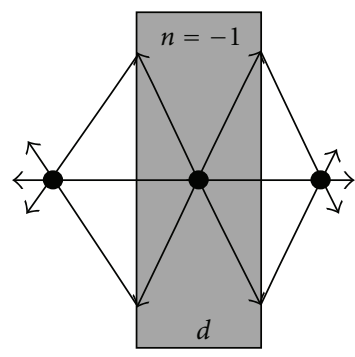

(d)
Figure 8: Ray tracing illustration of the flat lens made of an isotropic homogeneous medium with index of refraction $n=-1$. $d_{1}$ is the distance between the source and the first interface, and $d_{2}$ is the distance between the image point and the second interface, $d$ is the thickness of the slab. (a) $d_{1}=d / 2$, (b) $d_{1}>d / 2$, (c) $d_{1}<d / 2$, (d) source is placed inside of the slab.

imaging capability of a $\mathrm{PhC}$ slab with effective negative index of $n_{\text {eff }}=-1$ are shown in Figure 9. The imaging property is similar to that of the isotropic homogeneous medium with refractive index of $n=-1$. It is found that the image distance varies with the source distance in a similar way, with the sum of source distance, and image distance equals thickness of the $\mathrm{PhC}$ slab. On the other hand the transmission rate at the airPhC interfaces in this case is highly angular dependent [24]. Incident wave cannot couple completely to the $\mathrm{PhC}$. This is evident in Figure 9, where the field on the right-hand side of the slab is weaker than that on the left-hand side. From these simulations it can be concluded that the proposed $\mathrm{PhC}$ is not an isotropic homogeneous medium.

To examine the frequency dependence of effective negative index $n_{\text {eff }}$, we employ the FDTD method to simulate imaging of a point source of different frequency by a finitewidth $\mathrm{PhC}$ slab. The results shown in Figure 10 indicate that source of different frequency is focused to a different position. The point source locates at a fixed position in air, with a distance to the slab being half of the slab thickness. For the dispersive case of $\omega_{0}=0.315(2 \pi c / a)$ which corresponds to the aforementioned $n_{\text {eff }}=-1$, the image distance outside the slab is equal to the source distance as shown in Figure 10(a). For a lower frequency $\omega_{1}=0.300(2 \pi c / a)$ which corresponds to $n_{\text {eff }}<-1$, the image distance is slightly shorter than the source distance as shown in Figure 10(b). On the other hand, for a higher frequency $\omega_{2}=0.320(2 \pi c / a)$ which corresponds to $n_{\text {eff }}>-1$, the image distance is slightly longer than the source distance as shown in Figure 10(c). 


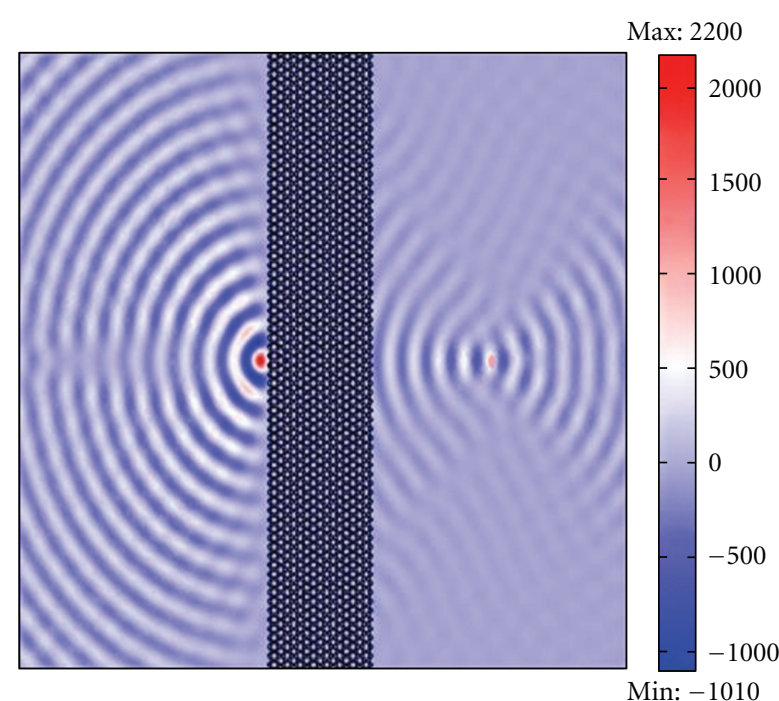

(a)

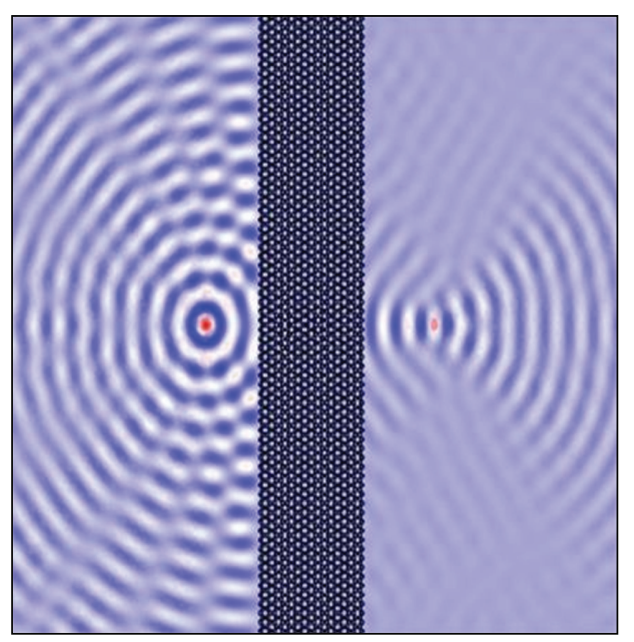

(b)

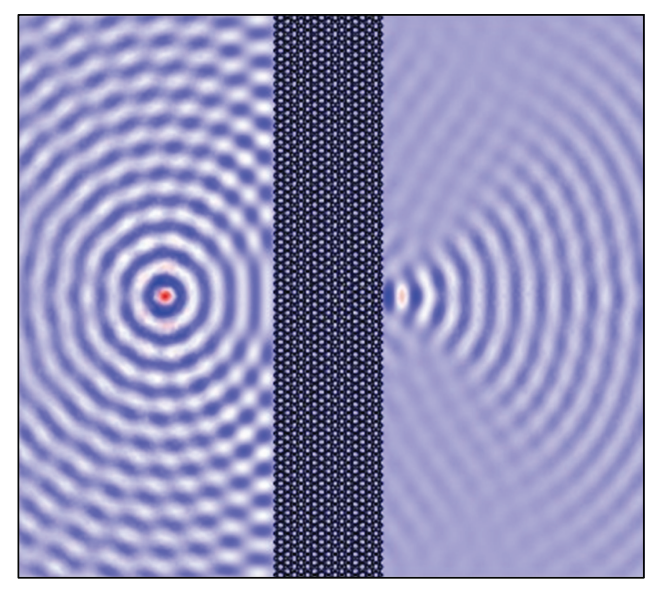

(c)

Max: 1800

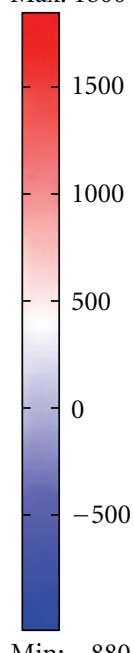

Min: -880

Max: 1700

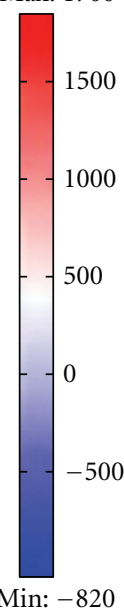

Min: -820

FIgure 9: Imaging of a point source by a slab of $2 \mathrm{D} \mathrm{PhC}$ of hexagonal lattice. The interface is normal to the $\Gamma-\mathrm{M}$ direction, and the normalized frequency is $\omega=0.315(2 \pi c / a)$. The distance between the source and the first interface is $d_{1}$, the distance between the image and the second interface is $d_{2}$, and the thickness of the slab is $d=8 \times \sqrt{3} a$. (a) $d_{1}<d / 2$, (b) $d_{1}=d / 2$, (c) $d_{1}>d / 2$.

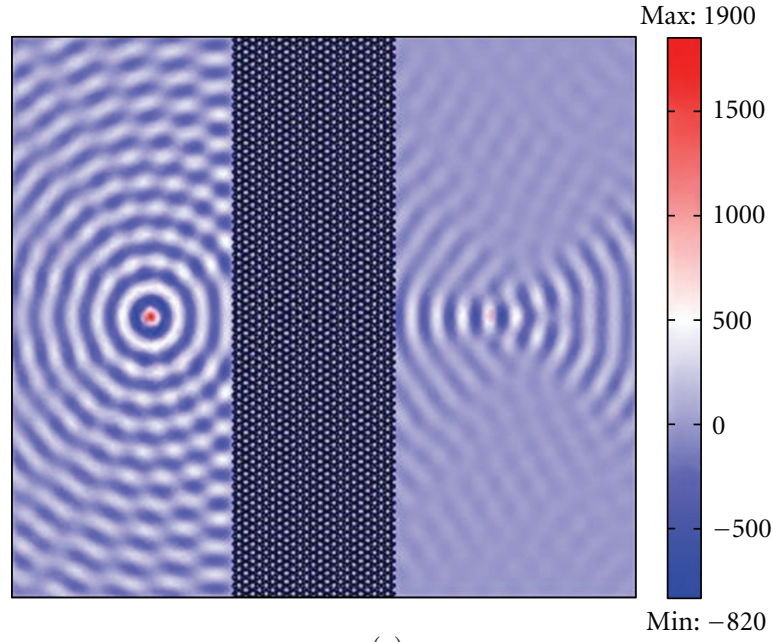

(a)

Max: 1800

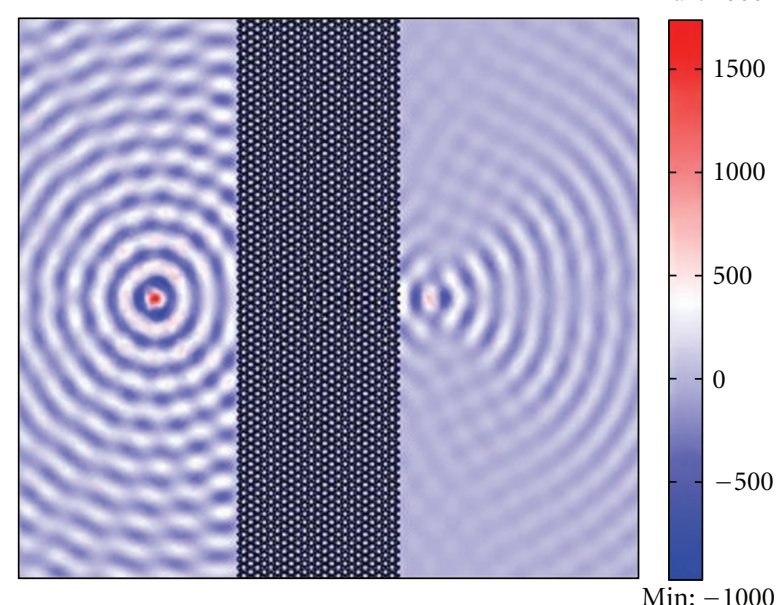

(b)

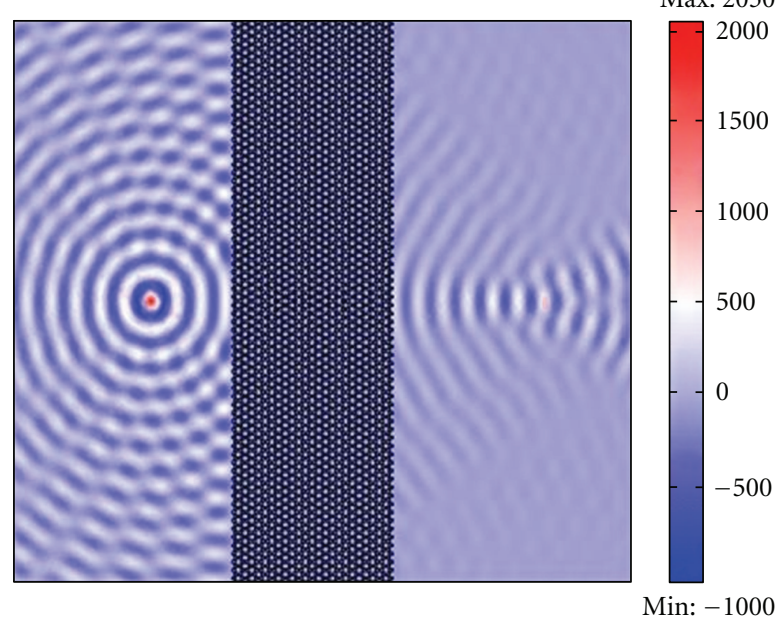

(c)

FIGURE 10: The FDTD simulations of point sources of three different frequencies placed in front of a $2 \mathrm{D} \mathrm{PhC} \mathrm{slab} \mathrm{of} \mathrm{hexagonal}$ lattices. The thickness of the slab is $d=12 \times \sqrt{3} a$, and the distance between source and the first slab interface is $d_{1}=d / 2$. The air-PhC interface is perpendicular to the $\Gamma-\mathrm{M}$ direction, and the normalized frequencies are, respectively, (a) $\omega=0.315(2 \pi c / a)$, (b) $\omega=0.300(2 \pi c / a)$, and (c) $\omega=0.320(2 \pi c / a)$. 


\section{Conclusions}

In this paper we demonstrated two types of negative refraction mechanisms of $\mathrm{PhC}$, namely the all-angle negative refraction without a negative index and the negative refraction with effective negative index. Light focusing effect occurs in both $\mathrm{PhC}$ slab systems. The AANR PhC slab can focus all Fourier components of an image within the frequency range of negative refraction. In the $\mathrm{PhC}$ with effective negative index, negative refraction has high angular dependence. The AANR PhC is more suitable for high-resolution superlensing application.

\section{Acknowledgments}

This work is supported by the National Natural Science Foundation of China under Grant no. 60588502. One of the authors (P. Jiang) would like to thank her parents and husband for understanding and spiritual support.

\section{References}

[1] S. Foteinopoulou, E. N. Economou, and C. M. Soukoulis, "Refraction in media with a negative refractive index," Physical Review Letters, vol. 90, no. 10, Article ID 107402, 4 pages, 2003.

[2] V. G. Veselago, "Photomagnetism," Soviet Physics-Uspekhi, vol. 10, p. 509, 1968.

[3] G. Gbur, H. F. Schouten, and T. D. Visser, "Achieving superresolution in near-field optical data readout systems using surface plasmons," Applied Physics Letters, vol. 87, no. 19, Article ID 191109, pp. 1-3, 2005.

[4] P. Markoš and C. M. Soukoulis, "Transmission studies of lefthanded materials," Physical Review B, vol. 65, no. 3, Article ID 033401, 4 pages, 2001.

[5] J. D. Wilson and Z. D. Schwartz, "Multifocal flat lens with lefthanded metamaterial," Applied Physics Letters, vol. 86, no. 2, Article ID 021113, 3 pages, 2005.

[6] M. Rajput, R. K. Sinha, and S. Rawal, "Negative refraction in visible region using nano-structured metallo-dielectric photonic crystal," in Photonic Fiber and Crystal Devices: Advances in Materials and Innovations in Device Applications III, vol. 7420 of Proceedings of SPIE, p. 74, San Diego, Calif, USA, August 2009.

[7] G. Sun, A. S. Jugessur, and A. G. Kirk, "Saturated superresolution imaging of photonic crystal with negative refraction," in Optical Data Storage, vol. 6282 of Proceedings of SPIE, Bellingham, Wash, USA, April 2006.

[8] X. Zhang, "Image resolution depending on slab thickness and object distance in a two-dimensional photonic-crystalbased superlens," Physical Review B, vol. 70, no. 19, Article ID 195110, 9 pages, 2004.

[9] Z. Li, B. Liang, H. Guo, J. Chen, and S. Zhuang, "Negative refraction and birefringence in a two-dimensional flat perfect photonic crystal," in Optoelectronic Materials and Devices II, vol. 6782 of Proceedings of SPIE, Wuhan, China, November 2007.

[10] S. Feng, Z. Y. Li, Z. F. Feng, B. Y. Cheng, and D. Z. Zhang, "Engineering the imaging properties of a metallic photoniccrystal slab lens," Applied Physics Letters, vol. 88, no. 3, Article ID 031104, 3 pages, 2006.
[11] S. Feng, Z. Y. Li, Z. F. Feng, K. Ren, B. Y. Cheng, and D. Z. Zhang, "Focusing properties of a rectangular-rod photoniccrystal slab," Journal of Applied Physics, vol. 98, no. 6, Article ID 063102, 6 pages, 2005.

[12] C. W. Wong, R. Chatterjee, K. Liu, C. J. Chen, and C. A. Husko, "Negative refraction and nonlinearities in photonic band gap nanostructures," in The International Society for Optical Engineering, vol. 6327 of Proceedings of SPIE, San Diego, Calif, USA, August 2006.

[13] S. Anantha Ramakrishna, "Physics of negative refractive index materials," Reports on Progress in Physics, vol. 68, no. 2, pp. 449-521, 2005.

[14] M. Notomi, "Negative refraction in photonic crystals," Optical and Quantum Electronics, vol. 34, no. 1-3, pp. 133-143, 2002.

[15] R. Moussa, S. Foteinopoulou, L. Zhang et al., "Negative refraction and superlens behavior in a two-dimensional photonic crystal," Physical Review B, vol. 71, no. 8, pp. 185106, 2005.

[16] E. Cubukcu, K. Aydin, E. Ozbay, S. Foteinopolou, and C. M. Soukoulis, "Subwavelength resolution in a two-dimensional photonic-crystal-based superlens," Physical Review Letters, vol. 91, no. 20, Article ID 207401, 4 pages, 2003.

[17] A. Berrier, M. Mulot, M. Swillo et al., "Negative refraction at infrared wavelengths in a two-dimensional photonic crystal," Physical Review Letters, vol. 93, no. 7, Article ID 10.1103/PhysRevLett.93.073902, 4 pages, 2004.

[18] X. Zhang, "Tunable non-near-field focus and imaging of an unpolarized electromagnetic wave," Physical Review B, vol. 71, no. 23, Article ID 235103, 5 pages, 2005.

[19] Z. Lu, C. Chen, C. A. Schuetz et al., "Subwavelength imaging by a flat cylindrical lens using optimized negative refraction," Applied Physics Letters, vol. 87, no. 9, Article ID 091907, 3 pages, 2005.

[20] C. Luo, S. G. Johnson, M. Soljačić, J. D. Joannopouls, and J. B. Pendry, "Novel optical phenomena with photonic crystals," in The International Society for Optical Engineering, vol. 5166 of Proceedings of SPIE, pp. 207-209, 2004.

[21] C. Luo, S. G. Johnson, J. D. Joannopoulos, and J. B. Pendry, "All-angle negative refraction without negative effective index," Physical Review B, vol. 65, no. 20, Article ID 201104, 4 pages, 2002.

[22] J. B. Pendry, "Negative refraction makes a perfect lens," Physical Review Letters, vol. 85, no. 18, pp. 3966-3969, 2000.

[23] L. Liu and S. He, "Near-field optical storage system using a solid immersion lens with a left-handed material slab," Optics Express, vol. 12, no. 20, pp. 4835-4840, 2004.

[24] Z. Ruan, Dispersion engineering: negative refraction and designed surface plasmons in periodic structures, Ph.D. thesis, Microelectronics and Applied Physics, Stockholm, Sweden, 2007. 


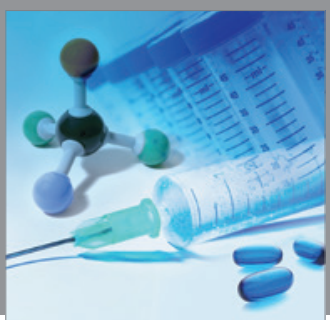

International Journal of

Medicinal Chemistry

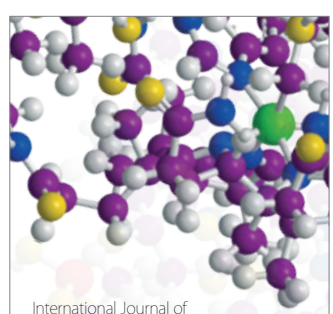

Carbohydrate Chemistry

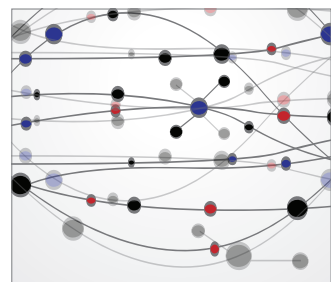

The Scientific World Journal
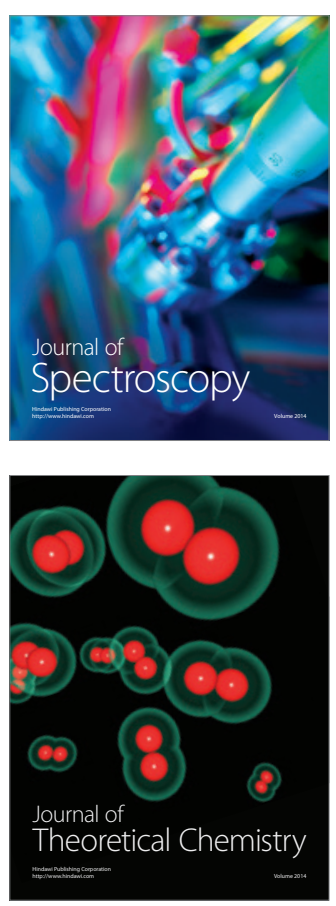
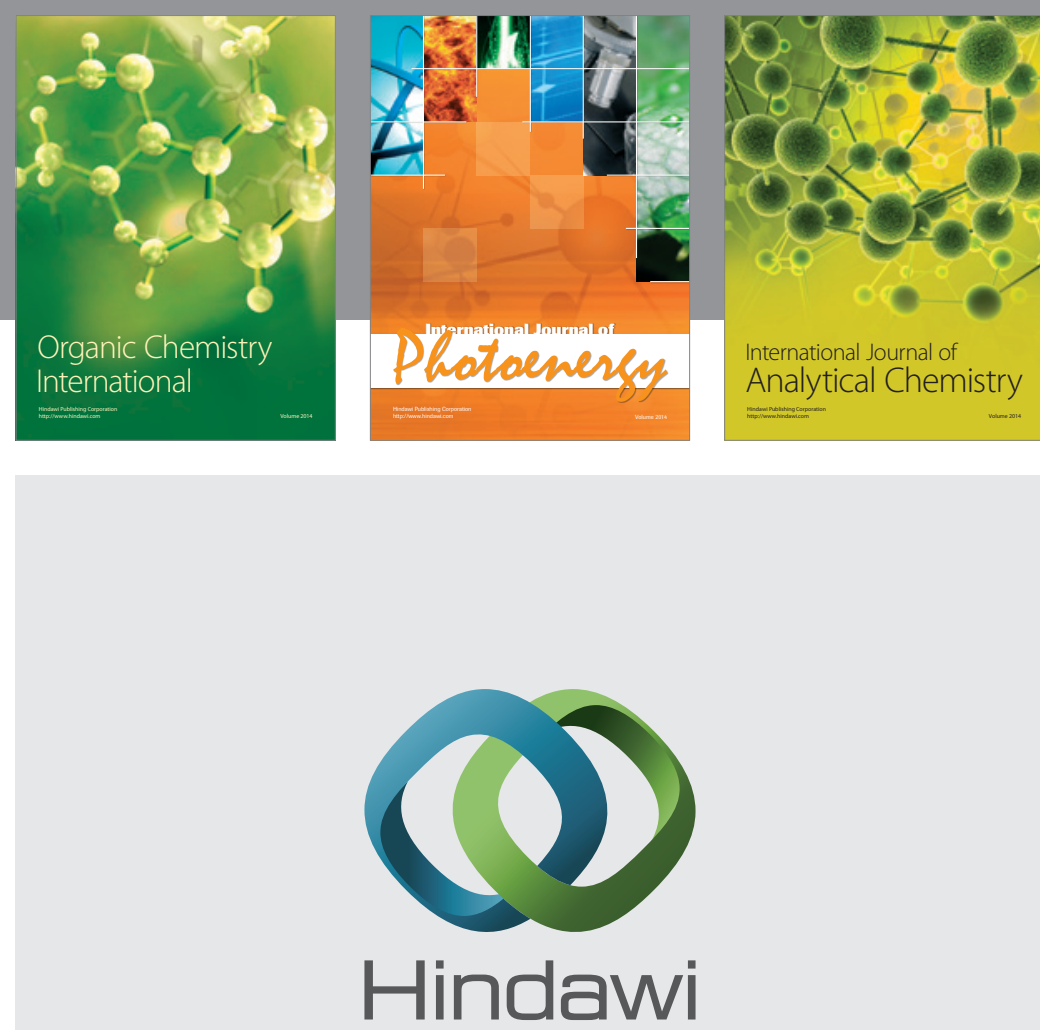

Submit your manuscripts at

http://www.hindawi.com
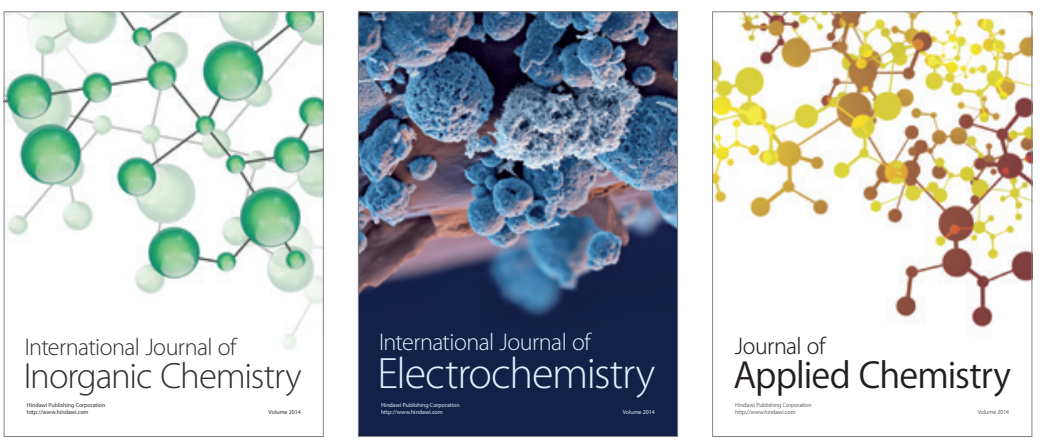

Journal of

Applied Chemistry
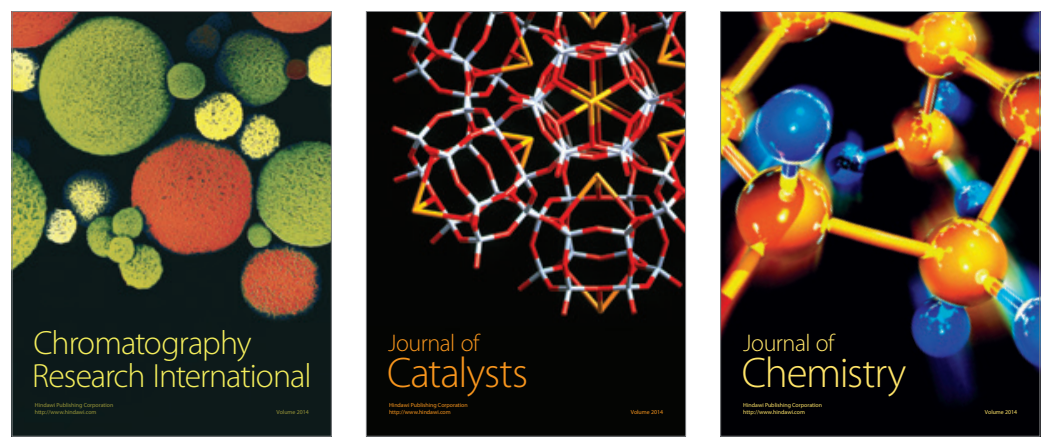
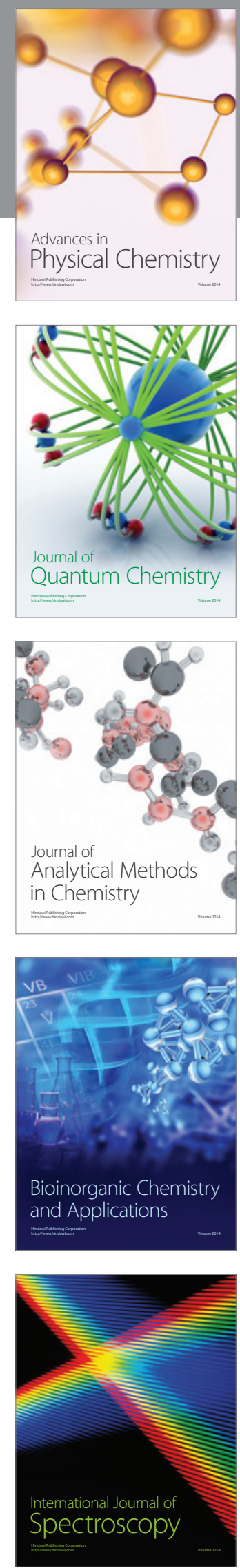